\title{
On Generalized Jacobsthal and Jacobsthal-Lucas polynomials
}

\author{
P. Catarino and M. L. Morgado
}

\begin{abstract}
In this paper we introduce a generalized Jacobsthal and JacobsthalLucas polynomials, $J_{h, n}$ and $j_{h, n}$, respectively, that consist on an extension of Jacobsthal's polynomials $J_{n}(x)$ and Jacobsthal-Lucas polynomials $j_{n}(x)$. We provide their properties and a generalization of the usual identities. We also present, for each one of these generalized polynomials, their ordinary generating functions and matrices. In the last part of the paper, we present some special kind of tridiagonal matrices whose entries are elements of these generalized polynomials.
\end{abstract}

\section{Introduction}

Fibonacci-like recursion relations are a special case of difference equations that can be solved by the combinatorics function technique method. Fibonacci polynomials are polynomials that can be defined by Fibonacci-like recursion relations and they were studied in 1883 by E. C. Catalan and E. Jacobsthal. For example, E. C. Catalan studied the polynomials $F_{n}(x)$ defined by the recurrence relation

$$
F_{n}(x)=x F_{n-1}(x)+F_{n-2}(x), n \geq 3
$$

Key Words: Jacobsthal polynomials, Lucas polynomials, generating function, generating matrix, Catalan's identity, d'Ocagne's identity.

2010 Mathematics Subject Classification: Primary 11B83, 11B37; Secondary 11B39, 11C20, 15B36.

Received: June, 2015.

Revised: July, 2015.

Accepted: July, 2015. 
where $F_{1}(x)=1$ and $F_{2}(x)=x$. This is an example of several polynomial sequences that can be defined by recurrence relations of order two.

Also many sequences of numbers can be defined by recurrence relations of order two, such as the Fibonacci numbers, Lucas numbers, Pell numbers, Pell-Lucas numbers, Jacobsthal numbers, Jacobsthal-Lucas numbers, among others. Various generalizations have also been studied (see, for example, $[1,3$, $4,8,16,17]$, among others researchers). In particular, for $n>0$, the sequence of Jacobsthal numbers $\left\{J_{n}\right\}_{n}$ is defined through the recurrence relation:

$$
J_{n+1}=J_{n}+2 J_{n-1}, \quad n \geq 1
$$

with $J_{0}=0$ and $J_{1}=1$. If we consider

$$
j_{n+1}=j_{n}+2 j_{n-1}, n \geq 1
$$

and let $j_{0}=2, j_{1}=1$, then we obtain the sequence of Jacobsthal-Lucas numbers $\left\{j_{n}\right\}_{n}$.

A generalization of this kind of sequence of numbers is the sequence $\left\{J_{k, n}\right\}_{n}$ of $k$-Jacobsthal numbers defined by

$$
J_{k, n+1}=k J_{k, n}+2 J_{k, n-1}, n \geq 1
$$

where $J_{k, 0}=0$ and $J_{k, 1}=1$. Also the sequence $\left\{j_{k, n}\right\}_{n}$ of $k$-Jacobsthal-Lucas numbers defined by

$$
j_{k, n+1}=k j_{k, n}+2 j_{k, n-1}, n \geq 1
$$

with $j_{k, 0}=2$ and $j_{k, 1}=1$ is a generalization of the Jacobsthal-Lucas sequence. Various studies have been published about some properties concerning this type of sequences, (see, for example, $[2,10,11]$ ). Note that in the particular case where $k=1,(4)$ reduces to (2) and (5) reduces to (3).

These numbers and their properties are useful in several areas as image processing, communications, signal processing, encoding (see [5, 14, 7, 19]), when certain linear systems of equations need to be solved, namely Toeplitz matrix problems. A recent example is the use of the circulant matrices to solve the Toeplitz structure linear system of equations obtained after the discretization of fractional differential equations (see [18]).

A natural extension of the Jacobsthal numbers is given by the Jacobsthal polynomials, which are introduced by Horadam in [12] and defined as follows:

$$
J_{n+1}(x)=J_{n}(x)+2 x J_{n-1}(x), \quad n>0, \quad J_{0}(x)=0, \quad J_{1}(x)=1 .
$$

Here we are interested in a generalization of (6), which we call the generalized Jacobsthal polynomials, or the $h(x)$-Jacobsthal polynomials, that are defined 
by:

$$
\begin{aligned}
J_{h, n+1}(x) & =J_{h, n}(x)+h(x) J_{h, n-1}(x), \quad n>0, \\
J_{h, 0}(x) & =0, \quad J_{h, 1}(x)=1,
\end{aligned}
$$

where $h(x)$ is a polynomial with real coefficients.

In a similar way, the Jacobsthal-Lucas polynomials, defined by

$$
j_{n+1}(x)=j_{n}(x)+2 x j_{n-1}(x), \quad n>0, \quad j_{0}(x)=2, \quad j_{1}(x)=1,
$$

are also a natural extension of the Jacobsthal-Lucas numbers. A generalization of (9) which we call the generalized Jacobsthal-Lucas polynomials, or the $h(x)$-Jacobsthal-Lucas polynomials is defined by

$$
\begin{aligned}
j_{h, n+1}(x) & =j_{h, n}(x)+h(x) j_{h, n-1}(x), \quad n>0, \\
j_{h, 0}(x) & =2, \quad j_{h, 1}(x)=1 .
\end{aligned}
$$

Note that in the particular case where $h(x)=2 x,(7)$ and (8) reduces to (6), (10) and (11) reduces to (9).

The paper is organized as follows: in Section 2 we present the generating functions for polynomials (7) and (8) and also (10) and (11). In section 3 we extend some usual known identities for these generalized polynomials. In section 4 we discuss their generating matrices and consider a special kind of tridiagonal matrices whose entries are both, generalized Jacobsthal and generalized Jacobsthal-Lucas polynomials. We end this paper with some conclusions and plans for further investigation.

\section{Generating functions}

In this section we present the ordinary generating function for polynomials (7) and (8) and also (10) and (11). Horadam in [11] present the ordinary generating function for sequences (2) and (3). Djordjević in [6] present the ordinary generating function for polynomials (6) and (9). In [13] we can find the ordinary generating function for sequence (4) and in [2] we have the ordinary generating function for sequence (5). Now, consider the ordinary generating function, $g_{J}(t)$, of the sequence $\left\{J_{h, n}(x)\right\}_{n}$ defined by:

$$
g_{J}(t)=\sum_{n=0}^{\infty} J_{h, n}(x) t^{n}
$$

Theorem 2.1. The ordinary generating function for the sequence $\left\{J_{h, n}(x)\right\}_{n}$ is

$$
g_{J}(t)=\frac{t}{1-t-t^{2} h(x)}
$$


Proof. Using (12), we have:

$$
\begin{aligned}
& g_{J}(t)-t g_{J}(t)-t^{2} h(x) g_{J}(t) \\
& =\sum_{n=0}^{\infty} J_{h, n}(x) t^{n}-\sum_{n=0}^{\infty} J_{h, n}(x) t^{n+1}-h(x) \sum_{n=0}^{\infty} J_{h, n}(x) t^{n+2} \\
& =\underbrace{J_{h, 0}(x)}_{0}+t \underbrace{J_{h, 1}(x)}_{1}+\sum_{n=2}^{\infty} J_{h, n}(x) t^{n}-\sum_{n=1}^{\infty} J_{h, n-1}(x) t^{n}-h(x) \sum_{n=2}^{\infty} J_{h, n-2}(x) t^{n} \\
& =t+\sum_{n=2}^{\infty} J_{h, n}(x) t^{n}-\underbrace{J_{h, 0}(x)}_{0} t-\sum_{n=2}^{\infty} J_{h, n-1}(x) t^{n}-h(x) \sum_{n=2}^{\infty} J_{h, n-2}(x) t^{n} \\
& =t+\sum_{n=2}^{\infty} J_{h, n}(x) t^{n}-\sum_{n=2}^{\infty} J_{h, n-1}(x) t^{n}-h(x) \sum_{n=2}^{\infty} J_{h, n-2}(x) t^{n} \\
& =t+\sum_{n=2}^{\infty} \underbrace{\left(J_{h, n}(x)-J_{h, n-1}(x)-h(x) J_{h, n-2}(x)\right)}_{0} t^{n}=t,
\end{aligned}
$$

and thus (13) follows.

The ordinary generating function, $g_{j}(t)$, of the sequence $\left\{j_{h, n}(x)\right\}$ is defined by:

$$
g_{j}(t)=\sum_{n=0}^{\infty} j_{h, n}(x) t^{n} .
$$

Theorem 2.2. The ordinary generating function for the sequence $\left\{j_{h, n}(x)\right\}_{n}$ is

$$
g_{j}(t)=\frac{2-t}{1-t-t^{2} h(x)}
$$

Proof. Using (14), we have:

$$
\begin{aligned}
g_{j}(t) & =j_{h, 0}(x) t^{0}+j_{h, 1}(x) t+\sum_{n=2}^{\infty} j_{h, n}(x) t^{n} \\
& =2+t+\sum_{n=2}^{\infty} j_{h, n}(x) t^{n} \\
& =2+t+\sum_{n=2}^{\infty}\left(j_{h, n-1}(x)+h(x) j_{h, n-2}(x)\right) t^{n}
\end{aligned}
$$




$$
\begin{aligned}
& =2+t+\sum_{n=2}^{\infty} j_{h, n-1}(x) t^{n}+h(x) \sum_{n=2}^{\infty} j_{h, n-2}(x) t^{n} \\
& =2+t+\sum_{n=2}^{\infty} j_{h, n-1}(x) t^{n-1} t+h(x) \sum_{n=2}^{\infty} j_{h, n-2}(x) t^{n-2} t^{2} \\
& =2+t+t\left(\sum_{n=1}^{\infty} j_{h, n-1}(x) t^{n-1}-j_{h, 0}(x)\right)+h(x) t^{2} \sum_{n=2}^{\infty} j_{h, n-2}(x) t^{n-2} \\
& =2+t-2 t+\sum_{n=0}^{\infty} j_{h, n}(x) t^{n}\left(t+h(x) t^{2}\right) \\
& =2-t+g_{j}(t)\left(t+h(x) t^{2}\right),
\end{aligned}
$$

and thus the result derives.

\section{Some identities for the generalized polynomials}

In this section we extend some usual known identities for these generalized polynomials. Similar identities for sequences (2), (3), (6), (9), (4) and (5) are also studied and we can find these identities in the literature (see, for example, [2], [6], [11], [12], [13], among others).

\subsection{The $h(x)-$ Jacobsthal polynomials}

The $h(x)$-Jacobsthal polynomials may be computed through the following Binet-style formula:

Theorem 3.1. (Generalized Binet-style formula)

For the sequence $\left\{J_{h, n}(x)\right\}_{n}$ we have

$$
J_{h, n}(x)=\frac{\alpha^{n}(x)-\beta^{n}(x)}{\alpha(x)-\beta(x)}, \quad n \geq 0,
$$

where

$$
\alpha(x)=\frac{1+\sqrt{1+4 h(x)}}{2} \quad \text { and } \quad \beta(x)=\frac{1-\sqrt{1+4 h(x)}}{2} .
$$

Proof. The characteristic equation of relation (7) is:

$$
\nu^{2}-\nu-h(x)=0,
$$

whose roots are $\alpha(x)$ and $\beta(x)$. Therefore, for $n \geq 0$, the solution of (7) is given by:

$$
J_{h, n}(x)=A \alpha^{n}(x)+B \beta^{n}(x) .
$$


From the initial conditions (8) it follows that $A$ and $B$ must satisfy the following system

$$
\left\{\begin{array}{l}
A+B=0 \\
A \alpha(x)+B \beta(x)=1
\end{array}\right.
$$

and hence $A=\frac{1}{\sqrt{1+4 h(x)}}$ and $B=\frac{-1}{\sqrt{1+4 h(x)}}$. Since $\alpha(x)-\beta(x)=\sqrt{1+4 h(x)}$, we obtain (17).

Next we generalize some identities known for the Jacobsthal polynomials, namely the Catalan, the Cassini and the d'Ocagne identities:

$$
\begin{aligned}
J_{n-r}(x) J_{n+r}(x)-J_{n}^{2}(x) & =(-1)^{n-r+1}(2 x)^{n-r} J_{r}(x), \\
J_{n-1}(x) J_{n+1}(x)-J_{n}^{2}(x) & =(-1)^{n}(2 x)^{n-1}, \\
J_{m}(x) J_{n+1}(x)-J_{m+1}(x) J_{h, n}(x) & =(-1)^{n}(2 x)^{n} J_{m-n}(x), \quad m>n,
\end{aligned}
$$

respectively. Before we proceed, let us first note that

$$
\alpha(x) \beta(x)=-h(x) .
$$

Theorem 3.2. (Generalized Catalan's identity)

For $n$ and $r$, nonnegative integer numbers, such that $r \leq n$, we have

$$
J_{h, n-r}(x) J_{h, n+r}(x)-J_{h, n}^{2}(x)=(-1)^{n-r+1}(h(x))^{n-r} J_{h, r}(x) .
$$

Proof. Using the generalized Binet-style formula (17), and taking (22) into account, we have

$$
\begin{aligned}
J_{h, n-r}(x) J_{h, n+r}(x)-J_{h, n}^{2}(x) & =\left(\frac{\alpha^{n-r}(x)-\beta^{n-r}(x)}{\alpha(x)-\beta(x)}\right)\left(\frac{\alpha^{n+r}(x)-\beta^{n+r}(x)}{\alpha(x)-\beta(x)}\right) \\
& -\left(\frac{\alpha^{n}(x)-\beta^{n}(x)}{\alpha(x)-\beta(x)}\right)^{2} \\
& =-(\alpha(x) \beta(x))^{n-r}\left(\frac{\alpha^{r}(x)-\beta^{r}(x)}{\alpha(x)-\beta(x)}\right)^{2} \\
& =(-1)^{n-r+1}(h(x))^{n-r} J_{h, r}(x) .
\end{aligned}
$$

In the particular case where $r=1$ we obtain the generalized Cassini identity.

Theorem 3.3. (Generalized Cassini's identity)

For any natural number $n$, we have

$$
J_{h, n-1}(x) J_{h, n+1}(x)-J_{h, n}^{2}(x)=(-1)^{n}(h(x))^{n-1} .
$$


Proof. This identity follows immediately by taking $r=1$ in (19) and taking the second initial condition in (8) into account.

Theorem 3.4. (Generalized d'Ocagne's identity)

Suppose that $n$ is a nonnegative integer number and $m$ any natural number. If $m>n$ then:

$$
J_{h, m}(x) J_{h, n+1}(x)-J_{h, m+1}(x) J_{h, n}(x)=(-1)^{n}(h(x))^{n} J_{h, m-n}(x) .
$$

Proof.

$$
\begin{aligned}
& J_{h, m}(x) J_{h, n+1}(x)-J_{h, m+1}(x) J_{h, n}(x)= \\
= & \left(\frac{\alpha^{m}(x)-\beta^{m}(x)}{\alpha(x)-\beta(x)}\right)\left(\frac{\alpha^{n+1}(x)-\beta^{n+1}(x)}{\alpha(x)-\beta(x)}\right) \\
- & \left(\frac{\alpha^{m+1}(x)-\beta^{m+1}(x)}{\alpha(x)-\beta(x)}\right)\left(\frac{\alpha^{n}(x)-\beta^{n}(x)}{\alpha(x)-\beta(x)}\right) \\
= & (\alpha(x) \beta(x))^{n}(\alpha(x)-\beta(x))\left(\frac{\alpha^{m-n}(x)-\beta^{m-n}(x)}{\left(\alpha(x)-\beta(x)^{2}\right)}\right) \\
= & (-h(x))^{n} J_{h, m-n}(x) .
\end{aligned}
$$

Note that if we consider $h(x)=2 x,(23),(24)$ and $(25)$ reduce to $(19),(20)$ and (21), respectively.

\subsection{The $h(x)$-Jacobsthal-Lucas polynomials}

The $h(x)$-Jacobsthal-Lucas polynomials may also be computed through the following Binet-style formula:

Theorem 3.5. (Generalized Binet's formula)

For the sequence $\left\{j_{h, n}(x)\right\}_{n}$ we have

$$
j_{h, n}(x)=\alpha^{n}(x)+\beta^{n}(x), \quad n \geq 0,
$$

where $\alpha(x)$ and $\beta(x)$ are defined as in (18).

Proof. Using again the characteristic equation of relation (10), which is the same of the relation (7), we have:

$$
j_{h, n}(x)=A \alpha^{n}(x)+B \beta^{n}(x),
$$


where $\alpha(x)$ and $\beta(x)$ are the roots of the characteristic equation. From the initial conditions (11):

$$
\left\{\begin{array}{l}
A+B=2 \\
A \alpha(x)+B \beta(x)=1
\end{array},\right.
$$

we get $A=B=1$ and then we obtain the required result.

Next, we obtain the usual identities.

Theorem 3.6. (Generalized Catalan's Identity)

For $n$ and $r$, nonnegative integer numbers, such that $r \leq n$, we have

$$
j_{h, n-r}(x) j_{h, n+r}(x)-j_{h, n}^{2}(x)=(-h(x))^{n-r}\left(j_{h, r}^{2}(x)-4(-h(x))^{r}\right) .
$$

Proof. Using the generalized Binet-style formula (26), doing some calculations and from the fact that $\alpha(x) \beta(x)=-h(x)$, we obtain:

$$
\begin{aligned}
j_{h, n-r}(x) j_{h, n+r}(x)-j_{h, n}^{2}(x) & =\left(\alpha^{n-r}(x)+\beta^{n-r}(x)\right)\left(\alpha^{n+r}(x)+\beta^{n+r}(x)\right) \\
- & \left(\alpha^{n}(x)+\beta^{n}(x)\right)^{2} \\
& =\alpha^{n}(x) \beta^{n}(x)\left(\frac{\beta^{r}(x)}{\alpha^{r}(x)}+\frac{\alpha^{r}(x)}{\beta^{r}(x)}-2\right) \\
& =(-h(x))^{n} \frac{\beta^{2 r}(x)+\alpha^{2 r}(x)-2 \alpha^{r}(x) \beta^{r}(x)}{\alpha^{r}(x) \beta^{r}(x)} \\
& =(-h(x))^{n-r}\left(\left(\alpha^{r}(x)+\beta^{r}(x)\right)^{2}-4(-h(x))^{r}\right) \\
& =(-h(x))^{n-r}\left(\left(j_{h, r}(x)\right)^{2}-4(-h(x))^{r}\right)
\end{aligned}
$$

In the case where $r=1$, we obtain the generalized Cassini identity, which in this case is given by:

\section{Theorem 3.7. (Generalized Cassini's identity)}

For any natural number $n$, we have

$$
j_{h, n-1}(x) j_{h, n+1}(x)-j_{h, n}^{2}(x)=(-1)^{n-1}(h(x))^{n-1}(1+4 h(x)) .
$$

Proof. This identity follows immediately by taking $r=1$ in the generalized Catalan identity and taking the second initial condition in (11) into account. 
Theorem 3.8. (Generalized d'Ocagne's identity)

Suppose that $n$ is a nonnegative integer number and $m$ any natural number. If $m>n$ then

$$
\begin{aligned}
& j_{h, m}(x) j_{h, n+1}(x)-j_{h, m+1}(x) j_{h, n}(x) \\
& =\sqrt{1+4 h(x)}(-h(x))^{n}\left(j_{h, m-n}(x)-2^{n-m+1}(1+\sqrt{1+4 h(x)})^{m-n}\right)
\end{aligned}
$$

Proof.

$$
\begin{aligned}
& j_{h, m}(x) j_{h, n+1}(x)-j_{h, m+1}(x) j_{h, n}(x)= \\
= & \left(\alpha^{m}(x)+\beta^{m}(x)\right)\left(\alpha^{n+1}(x)+\beta^{n+1}(x)\right)-\left(\alpha^{m+1}(x)+\beta^{m+1}(x)\right)\left(\alpha^{n}(x)+\beta^{n}(x)\right) \\
= & \alpha^{m}(x) \beta^{n}(x)(\beta(x)-\alpha(x))+\beta^{m}(x) \alpha^{n}(x)(\alpha(x)-\beta(x)) \\
= & (\alpha(x)-\beta(x))(\alpha(x) \beta(x))^{n}\left(\beta^{m-n}(x)-\alpha^{m-n}(x)\right) \\
= & (\alpha(x)-\beta(x))(\alpha(x) \beta(x))^{n}\left(\beta^{m-n}(x)+\alpha^{m-n}(x)-2 \alpha^{m-n}(x)\right) \\
= & \sqrt{1+4 h(x)}(-h(x))^{n}\left(j_{h, m-n}(x)-2^{n-m+1}(1+\sqrt{1+4 h(x)})^{m-n}(x)\right) .
\end{aligned}
$$

\section{The ordinary generating matrices}

A recurrent method for the study of the recurrence sequences is to define the so-called ordinary generating matrix. The $h(x)$-Jacobsthal polynomials and the $h(x)$-Jacobsthal-Lucas polynomials are two (among others) of the special case of a sequence which is defined recursively as a linear combination of the preceding $p$ terms:

$$
a_{n+p}=c_{0} a_{n}+c_{1} a_{n+1}+\ldots c_{p-1} a_{n+p-1},
$$

where $c_{0}, c_{1}, \ldots, c_{p-1}$ are real constants. Using the matrix method, consider a $p \times p$ matrix $B$, where the entries of the last row are the constants $c_{0}, c_{1}, \ldots, c_{p-1}$, the entries $b_{i, i+1}=1$ for $i=1, \ldots, p-1$ and the remaining entries are zero. Also define a matrix $A_{n}=\left(\begin{array}{llll}a_{n} & a_{n+1} & \ldots & a_{n+p-1}\end{array}\right)^{T}$ associated with (27), such that $B A_{n}=A_{n+1}$ and $A_{n}=B^{n} A_{0}$, where $A_{0}=$ $\left(\begin{array}{llll}a_{0} & a_{1} & \ldots & a_{p-1}\end{array}\right)^{T}$. Some results of Linear Algebra will be used in what follows with the aim to provide alternative expressions for the general term of the $h(x)$-Jacobsthal polynomials and the $h(x)$-Jacobsthal-Lucas polynomials. We start with the $h(x)$-Jacobsthal polynomials. 


\subsection{Generating matrix for the $h(x)$-Jacobsthal polynomials}

Using (7), (8) and (27), we have $p=2, c_{0}=h(x)$ and $c=1$. Hence, the associated matrix $B$ is, in this case, given by:

$$
B=B(x)=\left(\begin{array}{cc}
0 & 1 \\
h(x) & 1
\end{array}\right)
$$

with determinant $|B(x)|=-h(x)$.

Proposition 4.1. For all $n \geq 1$,

$$
B^{n}(x)=\left(\begin{array}{cc}
h(x) J_{h, n-1}(x) & J_{h, n}(x) \\
h(x) J_{h, n}(x) & J_{h, n+1}(x)
\end{array}\right) .
$$

Proof. By induction on $n$, we have:

- For $n=1, B^{1}(x)=\left(\begin{array}{ll}h(x) J_{h, 0}(x) & J_{h, 1}(x) \\ h(x) J_{h, 1}(x) & J_{h, 2}(x)\end{array}\right)=\left(\begin{array}{cc}0 & 1 \\ h(x) & 1\end{array}\right)$, and (28) is verified.

- Suppose now that (28) is true for $n$ and let us prove that it will be also true for $n+1$.

$$
\begin{aligned}
& B^{n+1}(x)=B(x) B^{n}(x)=\left(\begin{array}{cc}
0 & 1 \\
h(x) & 1
\end{array}\right)\left(\begin{array}{cc}
h(x) J_{h, n-1}(x) & J_{h, n}(x) \\
h(x) J_{h, n}(x) & J_{h, n+1}(x)
\end{array}\right) \\
& =\left(\begin{array}{cc}
h(x) J_{h, n}(x) & J_{h, n+1}(x) \\
h^{2}(x) J_{h, n-1}(x)+h(x) J_{h, n}(x) & h(x) J_{h, n}(x)+J_{h, n+1}(x)
\end{array}\right) \\
& =\left(\begin{array}{cc}
h(x) J_{h, n}(x) & J_{h, n+1}(x) \\
h(x)\left(h(x) J_{h, n-1}(x)+J_{h, n}(x)\right) & h(x) J_{h, n}(x)+J_{h, n+1}(x)
\end{array}\right) \\
& =\left(\begin{array}{cc}
h(x) J_{h, n}(x) & J_{h, n+1}(x) \\
h(x) J_{h, n+1}(x) & h(x) J_{h, n}(x)+J_{h, n+2}(x)
\end{array}\right) .
\end{aligned}
$$

Using the properties of the determinants of the matrices $B(x)$ and $B^{n}(x)$, we can deduce the Cassini identity in a different way than we have obtained in the previous section (see (24)).

Indeed, we know that $|B(x)|=-h(x)$ and $\left|B^{n}(x)\right|=h(x) J_{h, n-1}(x) J_{h, n+1}(x)-$ $h(x) J_{h, n}^{2}(x)$. Because $\left|B^{n}(x)\right|=|B(x)|^{n}$, the result follows immediately. 
Considering the eigenvalues of $B(x)$, it can be easily seen that two distinct eigenvalues can be determined: $\alpha(x)$ and $\beta(x)$ defined as in (18). The eigenvectors associated with $\alpha(x)$ and $\beta(x)$ are

$$
\left(\begin{array}{c}
a \\
\alpha(x) a
\end{array}\right), \quad\left(\begin{array}{c}
a \\
\beta(x) a
\end{array}\right)
$$

respectively, with $a \neq 0$. In particular, if $a=1$, we get the eigenvectors

$$
u_{1}=\left(\begin{array}{c}
1 \\
\alpha(x)
\end{array}\right), u_{2}=\left(\begin{array}{c}
1 \\
\beta(x)
\end{array}\right) .
$$

Writing

$$
A_{0}(x)=\left(\begin{array}{c}
J_{h, 0}(x) \\
J_{h, 1}(x)
\end{array}\right)=\left(\begin{array}{c}
0 \\
1
\end{array}\right)=\lambda_{1} u_{1}+\lambda_{2} u_{2},
$$

we obtain that $\lambda_{1}=\frac{1}{\alpha(x)-\beta(x)}$ and $\lambda_{2}=-\frac{1}{\alpha(x)-\beta(x)}$. Finally, applying $B^{n}(x)$, we get

$$
A_{n}(x)=B^{n}(x) A_{0}(x)=\left(\begin{array}{c}
J_{h, n}(x) \\
J_{h, n+1}(x)
\end{array}\right)
$$

which provides us an alternative way to achieve the generalized Binet-style formula for the generalized $h(x)$-Jacobsthal polynomials.

Following the ideas of [9], we know that the determinant of a special kind of tridiagonal matrices is related to a special $n t h$ order polynomial. If we consider the $(n \times n)$ tridiagonal matrices $M_{n}$, defined as:

$$
\left(\begin{array}{cccccccc}
a & b & & & & & & \\
c & d & e & & & & & \\
& c & d & e & & & & \\
& & & \ddots & \ddots & \ddots & & \\
& & & & & c & d & e \\
& & & & & & c & d
\end{array}\right)
$$

computing the sequence of determinants, we obtain:

$$
\begin{aligned}
\left|M_{1}\right| & =a \\
\left|M_{2}\right| & =d\left|M_{1}\right|-b c \\
\left|M_{3}\right| & =d\left|M_{2}\right|-c e\left|M_{1}\right| \\
\left|M_{4}\right| & =d\left|M_{3}\right|-c e\left|M_{2}\right| \\
& \vdots \\
\left|M_{n+1}\right| & =d\left|M_{n}\right|-c e\left|M_{n-1}\right|,
\end{aligned}
$$


and therefore we can easily obtain the following result:

Proposition 4.2. The $(n \times n)$ tridiagonal matrices

$$
J_{n}^{h}(x)=\left(\begin{array}{cccccccc}
1 & -1-h(x) & & & & & & \\
1 & 1 & -h(x) & & & & & \\
& 1 & 1 & -h(x) & & & & \\
& & & \ddots & \ddots & \ddots & & \\
& & & & & 1 & 1 & -h(x) \\
& & & & & & 1 & 1
\end{array}\right)
$$

satisfy

$$
\left|J_{n-1}^{h}(x)\right|=J_{h, n}(x)
$$

that is, the nth $h(x)$-Jacobsthal polynomial may be obtained through the computation of the the determinant of the $((n-1) \times(n-1))$ tridiagonal matrix $J_{n-1}^{h}(x)$.

Proof. In the particular case where $a=c=d=1, b=-1-h(x)$ and $e=-h(x)$ it is straightforward to see that the sequence of determinants $(30)$ becomes:

$$
\begin{aligned}
\left|M_{1}\right| & =\left|J_{1}^{h}(x)\right|=1=J_{h, 2}(x) \\
\left|M_{2}\right| & =\left|J_{2}^{h}(x)\right|=1+h(x)=J_{h, 3}(x) \\
\left|M_{3}\right| & =\left|J_{3}^{h}(x)\right|=1+2 h(x)=J_{h, 4}(x) \\
& \vdots \\
\left|M_{n-1}\right| & =\left|J_{n-1}^{h}(x)\right|=J_{h, n}(x)
\end{aligned}
$$

Another way of relating the $n t h$ order $h(x)$-Jacobsthal polynomial as the computation of a tridiagonal matrix, may be obtained following the ideas of [15], where the following result was presented:

Theorem 4.3. Let $\left\{x_{n}\right\}$ be any second order linear sequence, defined recursively as:

$$
x_{n+1}=A x_{n}+B x_{n-1}, \quad n \geq 1 \text {, }
$$


with $x_{0}=C, x_{1}=D$. Then, for all $n \geq 0$ :

$$
x_{n}=\left|\begin{array}{ccccccc}
C & D & 0 & 0 & \cdots & 0 & 0 \\
-1 & 0 & B & 0 & \cdots & 0 & 0 \\
0 & -1 & A & B & \cdots & 0 & 0 \\
\vdots & \vdots & \vdots & \ddots & \ddots & \vdots & \vdots \\
0 & 0 & 0 & 0 & \cdots & A & B \\
0 & 0 & 0 & 0 & \cdots & -1 & A
\end{array}\right|_{(n+1) \times(n+1)}
$$

In the case of the $h(x)$-Jacobsthal polynomials sequence, we have $A=1$, $B=h(x), C=0$ and $D=1$, and then, a direct application of Theorem 4.3 leads to the following proposition:

Proposition 4.4. For $n \geq 0$, we have

$$
J_{h, n}(x)=\left|\begin{array}{ccccccc}
0 & 1 & 0 & 0 & \cdots & 0 & 0 \\
-1 & 0 & h(x) & 0 & \cdots & 0 & 0 \\
0 & -1 & 1 & h(x) & \cdots & 0 & 0 \\
\vdots & \vdots & \vdots & \ddots & \ddots & \vdots & \vdots \\
0 & 0 & 0 & 0 & \cdots & 1 & h(x) \\
0 & 0 & 0 & 0 & \cdots & -1 & 1
\end{array}\right|_{(n+1) \times(n+1)}
$$

4.2 Generating matrix for the $h(x)$-Jacobsthal-Lucas polynomials

Following the same idea used for the $h(x)$-Jacobsthal polynomials, in the case of the $h(x)$-Jacobsthal-Lucas polynomials, we have $p=2, c_{0}=h(x)$ and $c_{1}=1$ in (27). The associated matrix $C(x)$ is given by:

$$
C=C(x)=\left(\begin{array}{cc}
0 & 1 \\
h(x) & 1
\end{array}\right)
$$

with $|C(x)|=-h(x)$ and $C(x)=B(x)$.

Before the statement of all powers of $C(x)$, we present the following result which provides us a way to obtain the $n t h h(x)$-Jacobsthal polynomial in terms of the $(n+1)$ th and $(n-1)$ th $h(x)$-Jacobsthal-Lucas polynomials.

Lemma 4.5. For all $n \geq 1$,

$$
(1+4 h(x)) J_{h, n}(x)=j_{h, n+1}(x)+h(x) j_{h, n-1}(x) .
$$


Proof. Using the fact that $1+4 h(x)=(\alpha(x)-\beta(x))^{2}, h(x)=-\alpha(x) \beta(x)$ and Binet-style formulas, we easily obtain:

$$
\begin{aligned}
(1+4 h(x)) J_{h, n}(x) & =(\alpha(x)-\beta(x))^{2}\left(\frac{\alpha^{n}(x)-\beta^{n}(x)}{\alpha(x)-\beta(x)}\right) \\
& =(\alpha(x)-\beta(x))\left(\alpha^{n}(x)-\beta^{n}(x)\right) \\
& =\left(\alpha^{n+1}(x)+\beta^{n+1}(x)\right)-\alpha(x) \beta(x)\left(\alpha^{n-1}(x)+\beta^{n-1}(x)\right) \\
& =j_{h, n+1}(x)+h(x) j_{h, n-1}(x) .
\end{aligned}
$$

Using (33), (10) and (11) in the entries of (28), we can conclude the following result.

Proposition 4.6. For all $n \geq 1$ :

$$
C^{n}(x)=\left(\begin{array}{cc}
h(x)\left(\frac{2 j_{h, n}(x)-j_{h, n-1}(x)}{1+4 h(x)}\right) & \frac{2 j_{h, n+1}(x)-j_{h, n}(x)}{1+4 h(x)} \\
h(x)\left(\frac{2 j_{h, n+1}(x)-j_{h, n}(x)}{1+4 h(x)}\right) & \frac{2 j_{h, n+2}(x)-j_{h, n+1}(x)}{1+4 h(x)}
\end{array}\right) .
$$

Proof. By induction on $n$ :

- Taking $n=1$ in (34), straightforward calculations lead to:

$$
\begin{aligned}
C(x) & =\left(\begin{array}{cc}
h(x)\left(\frac{2 j_{h, 1}(x)-j_{h, 0}(x)}{1+4 h(x)}\right) & \frac{2 j_{h, 2}(x)-j_{h, 1}(x)}{1+4 h(x)} \\
h(x)\left(\frac{2 j_{h, 2}(x)-j_{h, 1}(x)}{1+4 h(x)}\right) & \frac{2 j_{h, 3}(x)-j_{h, 2}(x)}{1+4 h(x)}
\end{array}\right) \\
& =\left(\begin{array}{cc}
0 & 1 \\
h(x) & 1
\end{array}\right) .
\end{aligned}
$$

Hence (34) holds for $n=1$.

- Assume that (34) is true for $n$. We shall show that (34) will also be true for $(n+1)$.

$$
\begin{aligned}
& C^{n+1}(x)=C(x) C^{n}(x) \\
& =\left(\begin{array}{cc}
0 & 1 \\
h(x) & 1
\end{array}\right)\left(\begin{array}{c}
h(x)\left(\frac{2 j_{h, n}(x)-j_{h, n-1}(x)}{1+4 h(x)}\right) \\
h(x)\left(\frac{2 j_{h, n+1}(x)-j_{h, n}(x)}{1+4 h(x)}\right)
\end{array}\right. \\
& \left.\begin{array}{cc}
\frac{2 j_{h, n+1}(x)-j_{h, n}(x)}{1+4 h(x)} \\
\frac{2 j_{h, n+2}(x)-j_{h, n+1}(x)}{1+4 h(x)}
\end{array}\right) \\
& =\left(\begin{array}{cc}
h(x)\left(\frac{2 j_{h, n+1}(x)-j_{h, n}(x)}{1+4 h(x)}\right) & \frac{2 j_{h, n+2}(x)-j_{h, n+1}(x)}{1+4 h(x)} \\
h(x)\left(\frac{2 j_{h, n+2}(x)-j_{h, n+1}(x)}{1+4 h(x)}\right) & \frac{2 j_{h, n+3}(x)-j_{h, n+2}(x)}{1+4 h(x)}
\end{array}\right),
\end{aligned}
$$

by (10), and therefore (34) is valid for $(n+1)$. 
Using the properties involving the determinants of the matrices $C(x)$ and $C^{n}(x)$ and doing some calculations we are able to obtain the generalized Cassini identity. From $\left|C^{n}(x)\right|=|C(x)|^{n}=(-h(x))^{n}$ we obtain:

$$
\begin{aligned}
& \frac{\left(2 j_{h, 1}(x)-j_{h, 0}(x)\right)\left(2 j_{h, n+2}(x)-j_{h, n+1}(x)\right)-\left(2 j_{h, n+1}(x)-j_{h, n}(x)\right)^{2}}{(1+4 h(x))^{2}}= \\
& =(-1)^{n}(h(x))^{n-1} \\
& \Leftrightarrow \frac{A}{(1+4 h(x))}=(-1)^{n}(h(x))^{n-1}(1+4 h(x)),
\end{aligned}
$$

where

$$
A=\left(2 j_{h, 1}(x)-j_{h, 0}(x)\right)\left(2 j_{h, n+2}(x)-j_{h, n+1}(x)\right)-\left(2 j_{h, n+1}(x)-j_{h, n}(x)\right)^{2} .
$$

Then it suffices to show that $A=-j_{h, n-1}(x) j_{h, n+1}(x)+j_{h, n}^{2}(x)$. Indeed

$$
\begin{aligned}
A & =\left(2 j_{h, 1}(x)-j_{h, 0}(x)\right)\left((1+2 h(x)) j_{h, n}(x)+h(x) j_{h, n-1}(x)\right)-\left(2 j_{h, n+1}(x)-j_{h, n}(x)\right)^{2} \\
& =(1+4 h(x))\left(j_{h, n}^{2}(x)-j_{h, n}(x) j_{h, n-1}(x)-h(x) j_{h, n-1}^{2}(x)\right) \\
& =(1+4 h(x))\left(j_{h, n}^{2}(x)-j_{h, n-1}(x)\left(j_{h, n}(x)+h(x) j_{h, n-1}(x)\right)\right) \\
& =(1+4 h(x))\left(j_{h, n}^{2}(x)-j_{h, n-1}(x) j_{h, n+1}(x)\right)
\end{aligned}
$$

obtaining, in this way an alternative for the derivation of the generalized Cassini identity.

As we have done in subsection 4.1, next we indicate how the $n t h$ order $h(x)$-Jacobsthal-Lucas polynomial can be obtained as the determinant of special tridiagonal matrices. Following the ideas of Proposition 4.2 it is easy to obtain the following result whose proof is then omitted.

Proposition 4.7. The $(n \times n)$ tridiagonal matrices

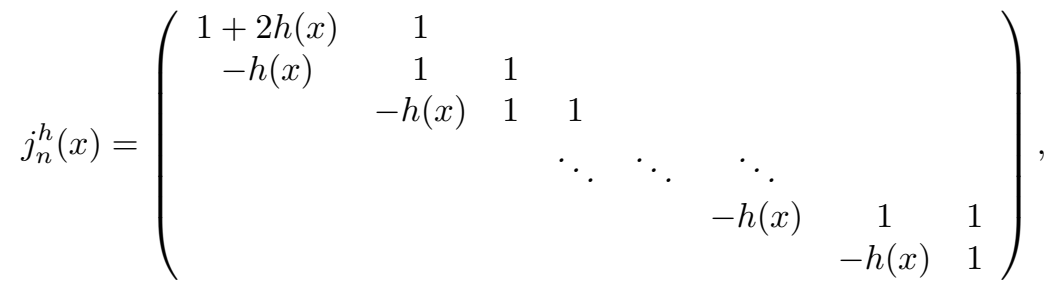

satisfy

$$
\left|j_{n-1}^{h}(x)\right|=j_{h, n}(x)
$$


that is, the nth $h(x)$ - Jacobsthal-Lucas polynomial may be obtained through the computation of the determinant of the $((n-1) \times(n-1))$ tridiagonal matrix $j_{n-1}^{h}(x)$.

Alternatively, we can use Theorem 4.3 with $A=1, B=h(x), C=2$ and $D=1$, to obtain:

Proposition 4.8. For $n \geq 0$, we have

$$
j_{h, n}(x)=\left|\begin{array}{ccccccc}
2 & 1 & 0 & 0 & \cdots & 0 & 0 \\
-1 & 0 & h(x) & 0 & \cdots & 0 & 0 \\
0 & -1 & 1 & h(x) & \cdots & 0 & 0 \\
\vdots & \vdots & \vdots & \ddots & \ddots & \vdots & \vdots \\
0 & 0 & 0 & 0 & \cdots & 1 & h(x) \\
0 & 0 & 0 & 0 & \cdots & -1 & 1
\end{array}\right|_{(n+1) \times(n+1)}
$$

\section{Conclusions}

Several generalizations of Fibonacci, Lucas, Pell, Jacobsthal, etc., numbers and polynomials can easily be found in the literature. Here we have considered an extension of the Jacobsthal and Jacobsthal-Lucas polynomials, by introducing a general polynomial function $h(x)$ which, in the case $h(x)=2 x$, reduces to the classical Jacobsthal and Jacobsthal-Lucas polynomials. For these classes of generalized polynomials we have provided their ordinary generating functions and matrices and we have also extended the usual known identities, as Catalan's, Cassini's and d'Ocagne's identities. As it can be easily observed, if we consider the particular case $h(x)=2 x$, all the ordinary generating functions and matrices and all the usual identities known to be valid for the classical Jacobsthal polynomials can be recovered.

In the future, we intend to study the circulant matrices associated with these generalized polynomials.

Acknowledgments. This work was supported by Portuguese Funds through FCT Fundação para a Cięncia e a Tecnologia, within the Project UID/MAT/00013/2013.

\section{References}

[1] C. Bolat and H. Köse, On the Properties of k-Fibonacci Numbers, Int. J. Contemp. Math. Sci. 22 (5) (2010), 1097-1105. 
[2] H. Campos, P. Catarino, A. P. Aires, P. Vasco and A. Borges, On some identities of $k$-Jacobsthal-Lucas Numbers, International Journal of Mathematical Analysis 8 (10) (2014), 489-494.

[3] P. Catarino, On some identities for $k$-Fibonacci sequence, Int. J. Contemp. Math. Sci. 9 (1) (2014), 37-42.

[4] P. Catarino, P. Vasco, A. Borges, H. Campos, A. P. Aires, Sums, Products and identities involving $k$-Fibonacci and $k$-Lucas sequences, JP J. Algebra Number Theory Appl. 32 (1) (2014), 63-77.

[5] P. J. Davis, Circulant Matrices, John Wiley \& Sons, New York, 1979.

[6] G. B. Djordjević, Generalized Jacobsthal polynomials, The Fibonacci Quarterly 38 (2000), 239-243.

[7] C. Erbas and M. M. Tanik, Generating solutions to the N-Queens problems using 2-circulants, Math. Mag. 68, 1995, 343-356.

[8] S. Falcón, On the k-Lucas numbers, Int. J. Contemp. Math. Sci. 6 (21) (2011), 1039-1050.

[9] S. Falcón, On the generating matrices of the $k$-Fibonacci numbers, Proyecciones Journal of Mathematics 32 (4) (2013) 347-357.

[10] A. F. Horadam, Jacobsthal and Pell Curves, The Fibonacci Quarterly 26 (1) (1988), 77-83.

[11] A. F. Horadam, Jacobsthal Representation Numbers, The Fibonacci Quarterly 34 (1) (1996), 40-53.

[12] A. F. Horadam, Jacobsthal Representation Polynomials, The Fibonacci Quarterly 35 (2) (1997), 137-148.

[13] D. Jhala, K. Sisodiya and G. P. S. Rathore, On some Identities for $k$-Jacobsthal Numbers, Int. Journal of Math. Analysis 7 (12) (2013), $551-556$.

[14] Z. L. Jiang and Z. X. Zhou, Circulant Matrices, Chengdu Technology University Publishing Company, Chengdu, 1999.

[15] E. Kilic, D. Tasci, and P. Haukkanen, On the generalized Lucas sequences by Hessenberg matrices, Ars Combin. 95 (2010), 383-395.

[16] T. Koshy, Fibonacci, Lucas and Pell numbers, and Pascal's triangle, Mathematical Spectrum 43 (3) (2011), 125-132. 
[17] Ayse Nalli and Pentti Haukkanen, On generalized Fibonacci and Lucas polynomials, Chaos, Solitons and Fractals 42 (2009) 3179-3186.

[18] W. Qu, S. L. Lei and S. W. Vong, Circulant and skew-circulant splitting iteration for fractional advection-diffusion equations, International Journal of Computer Mathematics, 91 (10) (2014), 2232-2242.

[19] Y. K. Wu and R. Z. Jia and Q. Li, g-circulant solutions to the $(0 ; 1)$ matrix equation $\mathrm{A}^{m}=\mathrm{J}_{n}^{*}$, Linear Algebra Appl. 345, 2002, 195-224.

Paula Catarino,

Mathematics Centre CMAT, Pole CMAT-UTAD,

Department of Mathematics,

University of Trás-os-Montes e Alto Douro, UTAD,

Quinta de Prados 5000-801, Vila Real, Portugal.

Email: pcatarin@utad.pt

Maria Luisa Morgado,

Mathematics Centre CMAT, Pole CMAT-UTAD,

Department of Mathematics,

University of Trás-os-Montes e Alto Douro, UTAD,

Quinta de Prados 5000-801, Vila Real, Portugal.

Email: luisam@utad.pt 